\title{
Study on the Effect of Temperature on Weigh-in-Motion Accuracy of Piezoelectric Thin
}

\author{
TANG Hao ${ }^{1, a}$ and DUAN Min ${ }^{1, b}$ \\ ${ }^{1}$ State Key Laboratory of Bridge Engineering Structural Dynamics, China Merchants Chongqing \\ Communications Technology Research \& Design Institute Co., Ltd., Chongqing 400067, China \\ atanghao9@163.com, bduanmin@cmhk.com
}

Keywords: traffic engineering; temperature compensation coefficient; least squares; weigh-in-motion; bridge safety

Abstract. Temperature is an important factor affecting the weighing accuracy of the piezoelectric thin film Weigh-in-Motion (WIM) system. Because the temperature compensation coefficient formula given in the existing technical data is not effective in practical application, and because the time and cost factors can not be used to calibrate the compensation coefficient of each temperature one by one, this paper studies the law between temperature and weigh-in-motion accuracy. The calibration coefficients were calculated by using the least-squares linear fitting method at two temperatures, and the compensation coefficients were calculated for all the temperature gradients. This not only reduced the test time and cost, but also to obtained a high weighing accuracy. The reliability and validity of the proposed method were proved by the experimental results and the actual test results.

\section{Introduction}

Vehicle load is a key fact affecting the safety and endurance of the bridges. And overload transportation is the major reason for bridge structure destruction and collapse. Research shows, vehicles are generally over load in practical. In order to suppress the damage of overload transportation on the infrastructures, European Union, American, Japan, Canada and other organizations have conducted a lot of researches on the Weigh-in-Motion(WIM) technology and obtained valuable achievements since 1950s. In 1974, American firstly utilized the WIM system in the research of vehicle loads. In the same year, France acquired a patent on piezoelectric WIM -the Vibracoax sensors [1,2]. In 1988, Britain developed a new and more advanced piezoelectric WIM sensor- Vibetek5, which is remodeled as Vibetek20 in 1991[3,4]. In 1992, FEHRI launched a plan called as COST323[5-7] according to the procedure framework of ECTD, the most important activities of which is the WIM system application test over a period of 30 mouths in Switzerland. In 1994, European Union started the Weighing in motion of Axle and Vehicles for Europe [8] plan. The systematical test for the product was conducted under the cold weather of Sweden from June, 1997 to June, 1998, which is known as the Cold Environment Test (CET). In 2000, the MSI company exhibited a copolymer piezoelectric sensor on the ITS annual meeting, which can measure the vehicle speed, number of axles and axle distance at the same time, as well as distinguish the vehicle's class and weigh them in motion. As so far, the piezoelectric WIM technology has been widely used over 60 countries, such as American, Brazil, Germany, Korea and so on in the past 10 years.

With the development of the safety monitoring technology of bridges, the bridge management and maintenance administrations pay more attention to the effect of traffic load on the bridge structure. It is expected that the service status of the bridges could be reflected scientifically through data as traffic flow and vehicle load, in conjunction with the traditional bridge structure safety monitoring information. Thus, proper measures can be made accordingly. The accuracy of the piezoelectric technology is mainly affected by the road surface roughness, vibrations and bounces of the vehicles and temperature. After installation The WIM system should be calibrated though the practical vehicle running experiments and temperature coefficient compensation. This is the key point to guarantee the accuracy of the system. Strictly speaking, different temperatures correspond to different temperature compensation coefficients. But in practical, it is hard to realize for the limitations of the 
implementation cost and time. This paper mainly investigates the relationship between the accuracy of the WIM system and the temperature as well as proposes a temperature coefficient calibration and verification method which is suitable for practical engineering use.

\section{Effect of temperature on WIM}

When the vehicle passing the piezoelectric sensors, the relationship between the static weight and dynamic weight of the vehicle is

$$
C=\frac{W_{0}}{W}
$$

Where, $W_{0}$ is the static weight. $W$ is the dynamic weight. $C$ is the calibration coefficient. For the WIM system, the key point is to inspect the consistency of the calibration coefficient $C$, namely, to test whether $C$ has good consistency when the same vehicle passing though the piezoelectric sensors with different speeds. Better consistency means higher accuracy. Wherein, the temperature has significant effect on $C$. The relationship between them is

$$
C=C_{0} \cdot C_{T}\left(T_{a}\right)
$$

Where, $C_{0}$ is the basic calibration coefficient. $C_{T}\left(T_{a}\right)$ is the temperature compensation coefficient at temperature $T_{a}$. The calibration coefficient is concluded from massive experiment data of different speeds and temperatures so as to obtain higher accuracy of WIM. However, it is impractical for the technicians to obtain the compensation coefficients of different temperatures one by one due to the limitation of cost and time.

Pater [9] points out that, the temperature will change the physicochemical properties of the glue used for encapsulation when embedding the piezoelectric sensors. Under different circumstances, the glue presents different properties of viscous, viscous-elastic or elastic, which affects the measurement signals reaching the piezoelectric sensors. Further, the accuracy of WIM will be affected. The formula for calculating the temperature compensation coefficient by paper [9] is

$$
C_{T}\left(T_{a}\right)=k_{1} \cdot 10^{w_{l}\left(T_{0}-T_{a}\right)}
$$

Where, $T_{a}$ is the road surface temperature in real measurement. $T_{0}$ is the initial temperature. $k_{1}$ and $w_{l}$ are calculation coefficients. Wherein, (1) $T_{0}=10, k_{1}=1.01286$ and $w_{l}=-0.005277$. Paper [10] uses the same formula as formula (3) for calculating the temperature compensation coefficient but with different parameters where (2) $T_{0}=45, k_{1}=3.8702, w_{l}=-0.0053$. Figure 1 shows the comparison of two curves under different temperature compensation coefficients, $30^{\circ} \mathrm{C}$ versus $100^{\circ}$ C. It can be seen that, the calculation results by formula (3) are inconsistent under the given two sets of parameters.

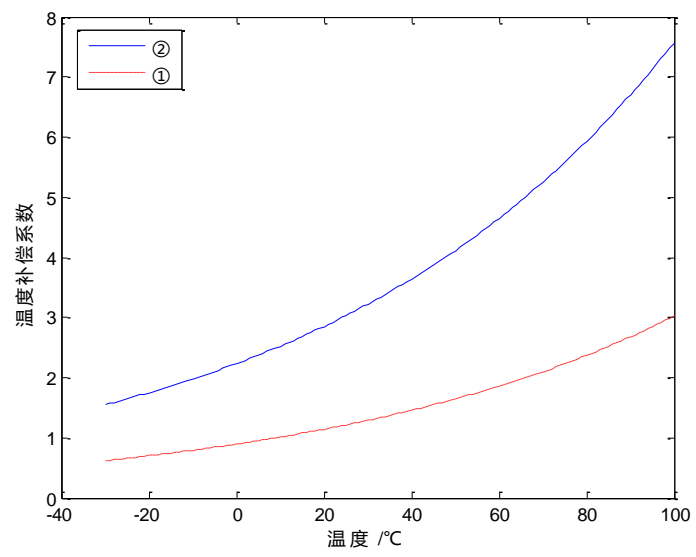

Fig.1 Temperature compensation coefficient at different temperature 


\section{Application of temperature compensation coefficient in engineering}

In 2016, a piezoelectric WIM system was installed on some expressway in China, shown as figure 2. Then vehicle running experiment was conducted to calibrate the parameters. A two-axle van was used in the experiment. The overall weight of the wan is $21.8 \mathrm{t}$ and the road surface temperature is 45 ${ }^{\circ} \mathrm{C}$. At last, the mean weight obtained by WIM was $21.11 \mathrm{t}$ when $C_{0}=380, C_{T}=218$. The mean test error calculated by formula (4) is $3.165 \%$.

$$
e=\left|\frac{y_{0}-y}{y_{0}}\right|
$$

Where $e$ is the test error. $y_{0}$ is the real weight. $y$ is the test weight.

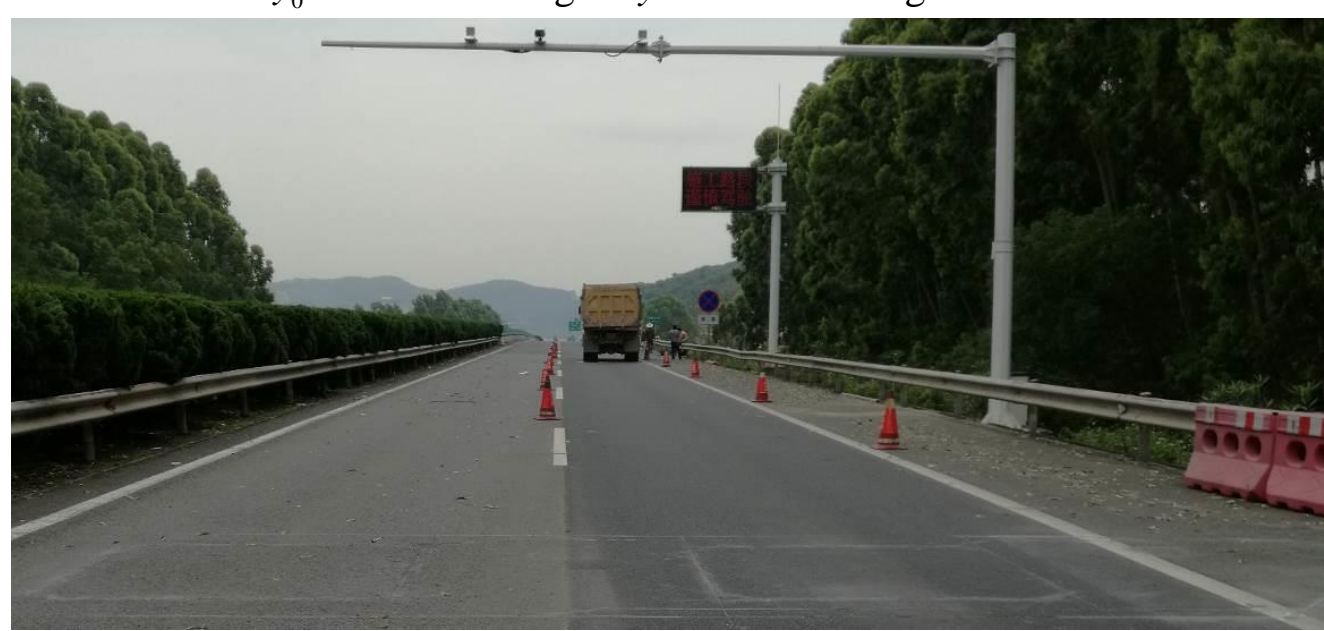

Fig.2 WIM system

According to the two temperature compensation curves calculated by the two sets of parameters (1), (2), the vehicle running experiment was conducted again under other temperatures. The test result is shown in table 1 . As it can be seen, the WIM error is two big at $36{ }^{\circ} \mathrm{C}, 30{ }^{\circ} \mathrm{C}$ and $26{ }^{\circ} \mathrm{C}$ no matter which set of parameter is used to calculate $C_{T}$. Referring to the WIM criterion, the overall weight error should be less than $10 \%$. Therefore, the method for calculating the temperature compensation coefficient given by formula (3) is not applicable in this situation. However, it is impossible to calibrate all temperatures in practical engineering for the limitations of cost and time. For example, the elapsed time for one vehicle-run of the mentioned experimental WIM system is 1.5 hours and cost of the car rental, road toll and labors is very high. Hence, it's necessary to develop a temperature compensation coefficient calculating method which suits for practical engineering applications and satisfies the requirements of WIM systems.

Table 1 Test results of different temperature

\begin{tabular}{|c|c|c|c|c|c|c|}
\hline Group & Real weight $(\mathrm{t})$ & Temperature $\left({ }^{\circ} \mathrm{C}\right)$ & $C_{0}$ & $C_{T}$ & Average test weight $(t)$ & Average test error $(\%)$ \\
\hline \multirow{4}{*}{ (1) } & \multirow{8}{*}{21.8} & 45 & \multirow{8}{*}{380} & 218 & 21.11 & 3.165 \\
\hline & & 36 & & 195.3561 & 14.64 & 32.844 \\
\hline & & 30 & & 181.5729 & 12.61 & 42.156 \\
\hline & & 26 & & 172.9935 & 10.03 & 53.991 \\
\hline \multirow{4}{*}{ (2) } & & 45 & & 218 & 21.11 & 3.165 \\
\hline & & 36 & & 195.3550 & 14.64 & 32.844 \\
\hline & & 30 & & 182.1173 & 12.65 & 41.973 \\
\hline & & 26 & & 172.8791 & 10.03 & 53.991 \\
\hline
\end{tabular}

\section{Temperature compensation checking method}

Shown as figure 3, a WIM experimental platform was built. And vehicle running experiments were conducted at the temperatures of $20^{\circ} \mathrm{C}$ and $13{ }^{\circ} \mathrm{C}$. The temperature compensation coefficient is calibrated by the rule of minimum mean test error and it yields $C_{T}(20)=12.69, C_{T}(13)=12.51$. The 
experiment results are show as table 2 . The mean test errors are $2.26 \%$ and $5.85 \%$ using the calibrated temperature, less than $10 \%$, satisfying the requirement of WIM system.

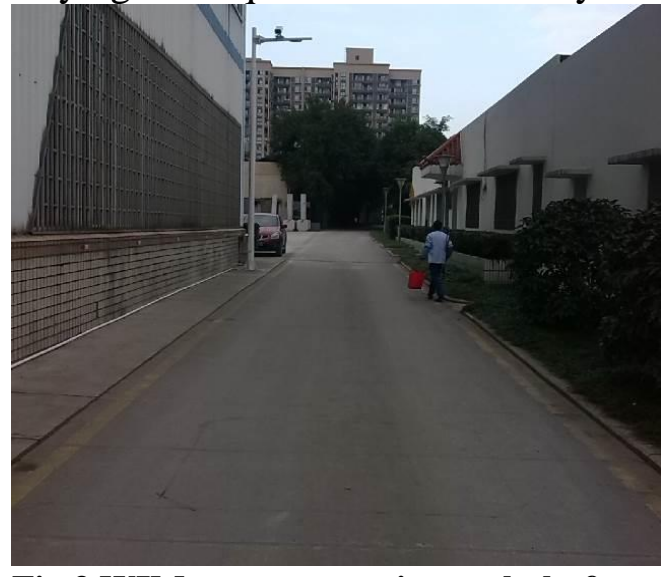

Fig.3 WIM system experimental platform

Table 2 Test results for two kinds of temperature

\begin{tabular}{|c|c|c|c|c|c|}
\hline \multirow{22}{*}{$\begin{array}{c}\text { Real weight } \\
10.87 \mathrm{t} \\
20^{\circ} \mathrm{C} \\
C_{T}=12.69\end{array}$} & Test weight (t) & Error (\%) & \multirow{22}{*}{$\begin{array}{c}\text { Real weight } \\
10.98 \mathrm{t} \\
13^{\circ} \mathrm{C} \\
C_{T}=12.51\end{array}$} & Test weight (t) & Error $(\%)$ \\
\hline & 10.66869 & 1.851954 & & 10.15243 & 7.537097 \\
\hline & 10.85365 & 0.150419 & & 10.04117 & 8.550369 \\
\hline & 10.60936 & 2.397751 & & 10.42829 & 5.024667 \\
\hline & 10.96787 & 0.900389 & & 10.57553 & 3.683699 \\
\hline & 11.09617 & 2.080643 & & 11.8338 & 7.775918 \\
\hline & 11.25202 & 3.514444 & & 11.92051 & 8.565704 \\
\hline & 10.81665 & 0.490796 & & 10.22972 & 6.83313 \\
\hline & 10.4573 & 3.796723 & & 12.22199 & 11.31138 \\
\hline & 10.56935 & 2.765848 & & 10.91467 & 0.594991 \\
\hline & 10.62297 & 2.272622 & & 10.78659 & 1.761463 \\
\hline & 11.16454 & 2.709666 & & 11.94789 & 8.814986 \\
\hline & 11.15322 & 2.605539 & & 11.18443 & 1.861856 \\
\hline & 10.55535 & 2.89466 & & 9.976122 & 9.142782 \\
\hline & 11.22184 & 3.236812 & & 10.19745 & 7.127016 \\
\hline & 10.61676 & 2.329703 & & 10.66567 & 2.862757 \\
\hline & 10.56522 & 2.803831 & & 12.3535 & 12.50912 \\
\hline & 10.61973 & 2.302406 & & 11.05805 & 0.710812 \\
\hline & 11.06161 & 1.762714 & & 10.77826 & 1.83737 \\
\hline & 10.98762 & 1.082038 & & 10.45982 & 4.737567 \\
\hline & 11.37517 & 4.647393 & & & \\
\hline & 10.96729 & 0.89501 & & & \\
\hline
\end{tabular}

Then the vehicle running data are applied to curve fitting using the least squares formula and it yields $C_{T}(30)=12.947$. Similarly, vehicle running experiment was conducted again under this temperature. The calculated mean test error is $3.902 \%$ by substituting the temperature compensation coefficient at $30^{\circ} \mathrm{C}$, shown as table 3 .

Table 3 Test results in $30^{\circ} \mathrm{C}$

\begin{tabular}{|c|c|c|c|c|}
\hline Real weight $(\mathrm{t})$ & Temperature $\left.^{\circ}{ }^{\circ} \mathrm{C}\right)$ & $C_{T}$ & Average test weight $(\mathrm{t})$ & Average test error $(\%)$ \\
\hline 11.02 & 30 & 12.947 & 10.59 & $3.902 \%$ \\
\hline
\end{tabular}

In conclusion, for the piezoelectric WIM system, vehicle running should be conducted at least under two different temperatures. The temperature compensation coefficients under different temperatures are calculated using the least squares fitting formula. This method guarantees the accuracy of WIM on condition of saving cost and time. Using this method for the calibration of the expressway WIM system mentioned in chapter 2, the temperature compensation coefficients at $45^{\circ}$ $\mathrm{C}$ and $36^{\circ} \mathrm{C}$ are obtained .The results are $C_{T}(45)=218$ and $C_{T}(36)=280$ with the mean test errors of $3.165 \%$ and $3.762 \%$, respectively. It can be calculated that $C_{T}(30)=321$ and $C_{T}(26)=349$. Then 
vehicle running experiment was conducted at the temperatures of $30^{\circ}$ Cand $26{ }^{\circ}$ Cto verify the accuracy of the method. The test results are shown in table 4 . Compared to table 1 , it can be found the proposed temperature compensation coefficient calculation method is more accurate with an error less than $10 \%$, proved to be reliable.

Table 4 Least squares calibration test results

\begin{tabular}{|c|c|c|c|c|c|}
\hline Real weight(t) & $\begin{array}{c}\text { Temperature }\left(^{\circ}\right. \\
\mathrm{C})\end{array}$ & $C_{0}$ & $C_{T}$ & Average test weight $(t)$ & Average test error(\%) \\
\hline \multirow{4}{*}{21.8} & 45 & \multirow{4}{*}{380} & 218 & 21.11 & 3.165 \\
\hline & 36 & & 280 & 20.98 & 3.762 \\
\hline & 30 & & 321 & 22.30 & 2.294 \\
\hline & 26 & & 349 & 20.24 & 7.156 \\
\hline
\end{tabular}

\section{Conclusions}

After the installation of the piezoelectric WIM system, calibration is needed to guarantee its accuracy. In practical engineering, it is impossible to calibrate all temperatures due to the limitations of cost and time. This paper proposes a method for calculating the temperature compensation coefficient using the least squares linear fitting algorithm. The method grantees the accuracy of WIM on condition of saving cost and time. At last, test results of the experimental platform and the practical engineering both prove the proposed method is reliable.

\section{Acknowledgements}

This work was financially supported by the Chongqing science and technology talent training program (cstc2014kjrc - qnrc30003) and the applied basic research project and information technology research project of Ministry of Transport of the People's Republic of China (2014319740160).

\section{References}

[1] G. Bailleul. Vibracoax ceramic piezoelectric traffic sensors[C]// National Traffic Data Acquisition Conference. New Mexico, 1996, 2: 596-611.

[2] P.M. Stewart. New sensors for axle detection and weigh-in-motion[C]// Road Traffic Monitoring, Second International Conference on. IET. 1989: 43-47.

[3] D.R. Fox, K. Sudworth, M.C. Booth, et al. A versatile piezoelectric cable[C]// IEEE 1986 Ultrasonics Symposium. IEEE, 1986: 599-602.

[4] D.R. Fox, K. Sudworth, M.C. Booth, et al. Vibetek 20*: a novel piezoelectric sensor cable[J]. Polymers in Defence, 1987: 5.

[5] B. Jacob, E.J. O’Brien. Weigh-in-motion: Recent developments in Europe[C]// 4th International Conference on Weigh-in-Motion-ICWIM4. Taipei, Taiwan, 2005.

[6] B. Jacob, E.J. O'Brien, S. Jehaes. Weigh-in-motion of road vehicles: final report of the COST 323 action[R]. Paris: Laboratoire Central des Ponts et Chaussées Publications, 2002.

[7] B. Jacob. Weigh-in-Motion of Road Vehicles, Proceedings of the Final Symposium of the Project WAVE, 1999[C]. WAVE Symposium, 1999.

[8] E.J. O'Brien, P. McNulty. Testing of bridge weigh-in-motion system in a sub-Arctic climate[J]. Journal of Testing and Evaluation, 2003, 31(6): 1-10.

[9] P. Burnos. Auto - calibration and temperature correction of WIM systems[C]// International Conference on Heavy Vehicles HVParis 2008. John Wiley \& Sons, Inc, 2013: 437-446.

[10] J. Gajda, R. Sroka, M. Stencel, et al. Multi - sensor weigh - in - motion system[C]// International Conference on Heavy Vehicles HVParis 2008. John Wiley \& Sons, Inc, 2008: 199-208. 